\title{
Contribuições e desafios da preceptoria nos Programas de Residência em
}

\section{Enfermagem}

\author{
Contributions and challenges of preceptorship in Nursing Residency Programs \\ Contribuciones y desafíos de la preceptoría en los Programas de Residencia en Enfermería
}

Recebido: 12/04/2021 | Revisado: 20/04/2021 | Aceito: 25/04/2021 | Publicado: 10/05/2021

Barbara Tavares de Paula Freitas

ORCID: https://orcid.org/0000-0003-4453-6838 Universidade Federal do Estado do Rio de Janeiro, Brasil E-mail: barbara.tavares82@gmail.com

Aline Coutinho Sento Sé

ORCID: https://orcid.org/0000-0001-9301-0379 Universidade Federal do Estado do Rio de Janeiro, Brasil E-mail: aline2506@hotmail.com

Raquel Calado da Silva Gonçalves

ORCID: https://orcid.org/0000-0003-0158-5031 Universidade Federal do Estado do Rio de Janeiro, Brasil E-mail: raquelcalado@yahoo.com.br

Gicélia Lombardo Pereira

ORCID: https://orcid.org/0000-0002-4032-2093 Universidade Federal do Estado do Rio de Janeiro, Brasil E-mail: gilombardo@ hotmail.com

\begin{abstract}
Resumo
Objetivo: Identificar a partir da busca na literatura científica as contribuições e desafios vivenciados pelos enfermeiros preceptores dos Programas de Residência em Enfermagem. Método: Estudo de revisão de literatura científica, realizado nas bases de dados do Sistema Online de Busca e Análise de Literatura Médica, Base de Dados de Enfermagem, Literatura Latino-Americana e do Caribe em Ciências da Saúde e Scientific Electronic Library Online, publicados entre 2010 e 2020 e nos idiomas português, inglês ou espanhol. Os resultados foram apresentados a partir da análise e síntese das evidências obtidas, discutindo-os à luz do referencial teórico. Resultados: A amostra final do estudo foi composta por oito artigos, de língua inglesa e portuguesa, predominantemente brasileiros, qualitativos e com ano de publicação em 2013, apontando como desafios à preceptoria nos Programas de Residência em Enfermagem carências relacionadas à qualificação, competências e reconhecimento profissional e como contribuições a participação ativa na formação dos alunos e busca constante de novos saberes para domínio teórico-prático. Considerações finais: Fazem-se necessárias mudanças capazes de estimular a satisfação e realização profissional, incentivo à qualificação pedagógica e reconhecimento da importância do papel do preceptor na formação dos residentes.
\end{abstract}

Palavras-chave: Educação de pós-graduação em enfermagem; Enfermagem; Ensino; Preceptoria; Internato e residência; Internato não médico; Residência não médica; Residência não odontológica.

\begin{abstract}
Objective: To identify from the search in the scientific literature, the contributions and challenges experienced by nurse preceptors of Nursing Residency Programs. Method: Study of scientific literature review, carried out in the databases of Online System for Search and Analysis of Medical Literature, Nursing Database, Latin American and Caribbean Literature in Health Sciences and Scientific Electronic Library Online, published between 2010 and 2020 and in Portuguese, English or Spanish. The results were presented based on the analysis and synthesis of the evidence obtained, discussing them in the light of the theoretical framework. Results: The final sample of the study consisted of eight articles in English and Portuguese, predominantly Brazilian, qualitative and with year of publication in 2013, pointing out challenges to preceptorship in Nursing Residency Programs shortages related to qualification, skills and professional recognition and as a contribution to active participation in the training of students and constant search for new knowledge for theoretical and practical domain. Final considerations: Changes are necessary to stimulate professional satisfaction and fulfillment, incentive to pedagogical qualification and recognition of the importance of the preceptor's role in the training of residents are necessary.
\end{abstract}

Keywords: Education nursing graduate; Nursing; Teaching; Preceptorship; Internship and residency; Internship nonmedical; Nonmedical residence; Nondental residence. 


\begin{abstract}
Resumen
Objetivo: Identificar a partir de la búsqueda en la literatura científica, los aportes y desafíos vividos por las enfermeras preceptoras de los Programas de Residencia en Enfermería. Método: Estudio de revisión de literatura científica, realizado en las bases de datos del Sistema en Línea de Búsqueda y Análisis de Literatura Médica, Base de Datos de Enfermería, Literatura Latinoamericana y del Caribe en Ciencias de la Salud y Biblioteca Electrónica Científica en Línea, publicada entre 2010 y 2020 y en portugués, inglés o español. Los resultados se presentaron a partir del análisis y síntesis de la evidencia obtenida, discutiéndolos a la luz del marco teórico. Resultados: La muestra final del estudio consistió en ocho artículos en inglés y portugués, predominantemente brasileños, cualitativos y con año de publicación en 2013, señalando desafíos a la preceptoría en la escasez de Programas de Residencia en Enfermería relacionados con la calificación, habilidades y reconocimiento profesional y como contribución a la participación en la formación de los estudiantes y búsqueda constante de nuevos conocimientos para el dominio teórico y práctico. Consideraciones finales: Son necesarios cambios para estimular la satisfacción y realización profesional, incentivar la calificación pedagógica y el reconocimiento de la importancia del rol del preceptor en la formación de los residentes.

Palabras clave: Educación de postgrado en enfermería; Enfermería; Ensenãnza; Preceptoría; Internado y residencia; Internado no médico; Residencia no médica; Residencia no dental.
\end{abstract}

\title{
1. Introdução
}

A residência na área da saúde é definida como um curso de Pós-graduação lato sensu, a qual contribui para a formação de profissionais nos campos da assistência, gestão e ensino (Martins, Caregnato, Barroso, \& Ribas, 2016). Caracterizada pelo aprofundamento científico e proficiência técnica decorrentes do treinamento em serviço, contempla conteúdos de natureza assistencial, educativa, administrativa e de investigação científica, de forma a atender às necessidades das populações e do perfil epidemiológico de cada região brasileira (Brasil, 1996; Fajardo, Rocha, \& Pasini, 2010; Silva et al., 2021).

Afirma-se que a residência em enfermagem teve origem nos Estados Unidos da América (EUA). No Brasil, foi implementada em 1961 pelo Hospital Infantil do Morumbi, na cidade de São Paulo, com o objetivo de aperfeiçoar a Enfermagem Pediátrica por meio de atividades teóricas e práticas (Martins et al., 2016; Fajardo et al., 2010), porém sem legislação regulamentadora à época.

Data de 1994 a realização de seminários pela Associação Brasileira de Enfermagem (ABEn) que impulsionaram a criação da Comissão Nacional de Residência em Enfermagem e de 1996, o Projeto de Lei no 2.264, que institui a Residência em Enfermagem na modalidade de Pós-graduação (Brasil, 1996; Fajardo et al., 2010).

Por meio da Resolução nº 259/2001, o Conselho Federal de Enfermagem delibera sobre o registro de especialista, na modalidade de Residência em Enfermagem, aos profissionais que atendam aos padrões estabelecidos na presente Resolução, como também abarca as áreas de conhecimento da Enfermagem, estrutura, planejamento pedagógico, constituição do corpo docente e/ou técnico profissional e tipos de instituições que poderão oferecer os Programas de Residência em Enfermagem (Brasil, 2001).

A busca por essa tipologia de especialização atrai, principalmente, recém-formados (Silva et al., 2021) por ter como essência o treinamento em situações reais de trabalho vinculado à discussão acadêmica, além de facilitar na transição de enfermeiro recém-graduado para especialista (Zanoni, Haddad, Rossaneis, Vannuchi, \& Gvozd, 2015).

Os programas de residência trabalham na perspectiva da preceptoria a partir do acompanhamento e da supervisão executados por profissionais do serviço de saúde (Antunes, Daher, \& Ferrari, 2017). A preceptoria deve ser realizada por enfermeiros em seu próprio cenário de trabalho, que precisam criar estratégias para executar as ações inerentes à rotina, administração e gerenciamento de demandas adicionais, concomitantemente ao acompanhamento dos residentes, inserindo-os no campo de aprendizado e apoiando-os no desenvolvimento de novas competências e habilidades (Ferreira, Dantas, \& Valente, 2018).

Ressalta-se que o preceptor é o profissional que participa ativamente do processo de formação do residente, fazendose imprescindível responsabilidade pedagógica, conhecimento teórico e destreza clínica, com vistas à aprendizagem 
significativa (Demogalski et al., 2021; Martins \& Scarcella, 2020). Ensinar, planejar ações educativas e estimular a reflexão crítica são aptidões não abordadas na graduação (Paczek \& Alexandre, 2019) e que podem se tornar desafios para os profissionais que necessitam de exercícios internos de autoaprendizagem (Ferreira, Dantas, \& Valente, 2018).

Assim, busca-se uma visão ampliada sobre os aspectos que envolvem a atividade de preceptoria na prática do ensino e do cuidado na perspectiva do enfermeiro preceptor, fundamentando-se na questão norteadora: quais são as contribuições e desafios vivenciados pelos enfermeiros preceptores dos Programas de Residência em Enfermagem? Diante disso, o objetivo desta pesquisa foi identificar a partir da busca na literatura científica as contribuições e desafios vivenciados pelos enfermeiros preceptores dos Programas de Residência em Enfermagem.

\section{Metodologia}

Estudo de revisão de literatura científica seguindo as etapas de identificação do problema de pesquisa, busca da literatura, avaliação dos dados, análise dos dados e apresentação dos resultados (Whittemore, 2005).

Definiu-se como problema de pesquisa: quais as contribuições e desafios vivenciados pelos enfermeiros preceptores dos Programas de Residência em Enfermagem?

Realizou-se busca da literatura no período de janeiro a fevereiro de 2020, nas bases de dados Sistema Online de Busca e Análise de Literatura Médica (MEDLINE), Base de Dados de Enfermagem (BDENF), Literatura Latino-Americana e do Caribe em Ciências da Saúde (LILACS) e Scientific Electronic Library Online (Scielo).

Efetivou-se a pesquisa com os descritores em ciências da saúde (DeCS) "Educação de pós-graduação em enfermagem", "Enfermagem", "Preceptor", "Internato e residência", "Internato não médico", "Residência não médica" e "Residência não odontológica", utilizando-se o operador booleano "AND", através das seguintes combinações: "Educação de pós-graduação em enfermagem AND Enfermagem AND Preceptor"; "Educação de pós-graduação em enfermagem AND Enfermagem AND Preceptor AND Internato e residência"; "Educação de pós-graduação em enfermagem AND Enfermagem AND Preceptor AND Residência não médica"; "Educação de pós-graduação em enfermagem AND Enfermagem AND Preceptor AND Internato não médico"; "Enfermagem AND Preceptor AND Residência não médica AND Internato não médico"; "Enfermagem AND Preceptor AND Residência não médica AND Residência não odontológica"; "Enfermagem AND Preceptor AND Internato e residência"; "Enfermagem AND Preceptor AND Internato não médico"; e "Enfermagem AND Preceptor AND Residência não médica".

Consistiram-se como critérios de inclusão para o estudo artigos disponíveis na íntegra, publicados entre 2010 e 2020 e nos idiomas português, inglês ou espanhol. Os critérios de exclusão foram artigos que, após leitura, não contemplavam o objeto de estudo e artigos duplicados.

Iniciou-se a avaliação dos dados com base na leitura dos títulos e resumos para exclusão dos materiais duplicados e que não correspondiam ao objetivo do estudo, assim como recuperação dos textos na íntegra.

Procedeu-se a análise dos dados através da leitura exaustiva dos artigos que compuseram a amostra em busca das contribuições e desafios dos preceptores nos Programas de Residência em Enfermagem e utilização de instrumento de coleta desenvolvido pelas autoras, para fins deste estudo, contendo: caracterização segundo autor, local e ano de publicação, título, tipo de estudo, idioma, qualis, tema, objetivo e principais resultados.

Apresentaram-se os resultados a partir da análise e síntese das evidências obtidas nos estudos, discutindo-os à luz do referencial teórico.

\section{Resultados}

O levantamento bibliográfico realizado através dos descritores "Educação de pós-graduação em enfermagem", 
"Enfermagem", "Preceptor", "Internato e residência", "Internato não médico", "Residência não médica" e "Residência não odontológica" e das combinações já descritas revelaram um total de 919 publicações, sendo 862 na MEDLINE, 24 na BDENF, 24 na LILACS e nove na Scielo. Compreendendo o momento da pré-análise, as 919 publicações foram submetidas aos critérios de exclusão previamente elencados.

Definiu-se, após a leitura dos títulos e resumos, a exclusão de 889 artigos, por se tratar de textos indisponíveis, duplicados, não abordarem o objeto de pesquisa, publicações anteriores ao ano de 2010 e idiomas que não fossem português, inglês ou espanhol. Desta forma, restaram, 30 artigos para a leitura completa.

Num segundo momento, todos os 30 artigos selecionados foram lidos com o objetivo de explorar o material e compreender a real aproximação com o objeto de pesquisa, sendo excluídas 22 publicações. Assim, identificaram-se oito publicações, as quais compuseram a amostra final deste estudo, conforme a Figura 1.

Figura 1 - Fluxograma de seleção dos artigos para revisão. Rio de Janeiro, RJ, Brasil, 2020.
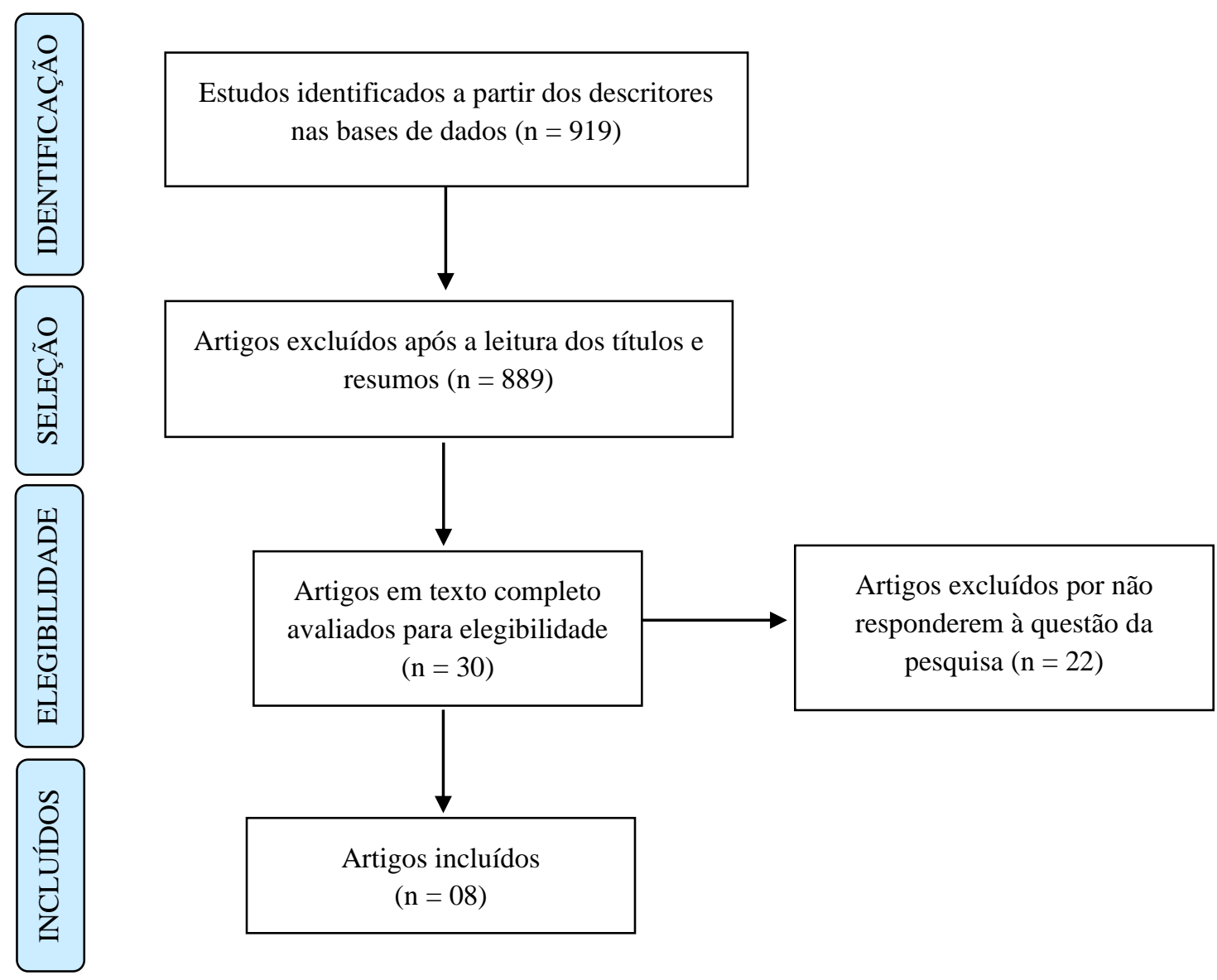

Fonte: Autores (2020).

Dos artigos que compuseram o corpus desta revisão, quatro foram publicados na língua inglesa (Jarnulf, Skytt, Martensson, \& Engstrom, 2019; Delack, Martin, McCarthy, \& Sperhac, 2015; Wiseman, 2013; e Haggerty, Holloway, \& Wilson, 2013), um na língua inglesa e portuguesa (Filho \& Santos, 2018) e três na língua portuguesa (Antunes et al., 2017; Ribeiro \& Prado, 2013; e Barbeiro, Miranda, \& Souza, 2010). Desta amostra, quatro produções estavam indexadas na base de dados MEDLINE (Jarnulf et al., 2019; Delack et al., 2015; Wiseman, 2013; e Haggerty et al., 2013), duas na BDENF (Antunes et al., 2017; e Barbeiro et al., 2010), uma na LILACS (Filho \& Santos, 2018) e uma na Scielo (Ribeiro \& Prado, 2013). 
Em relação à distribuição geográfica, quatro estudos (50\%) foram realizados no Brasil (Filho \& Santos, 2018; Antunes et al., 2017; Ribeiro \& Prado, 2013; e Barbeiro et al., 2010); dois (25\%) nos EUA (Delack et al., 2015; e Wiseman, 2013); um (12,5\%) na Suécia (Jarnulf et al., 2019) e um (12,5\%) na Nova Zelândia (Haggerty et al., 2013).

Quanto ao ano de publicação o mais prevalente foi 2013 com duas publicações (Wiseman, 2013, e Haggerty et al., 2013), seguido de uma em 2019 (Jarnulf et al., 2019), uma em 2018 (Filho \& Santos, 2018), uma em 2017 (Antunes et al., 2017), uma em 2015 (Delack et al., 2015), uma em 2013 (Ribeiro \& Prado, 2014) e uma em 2010 (Barbeiro et al., 2010).

O Quadro 1 apresenta um resumo das publicações selecionadas, caracterizadas quanto a autoria, local, ano de publicação, título, tipo de estudo, idioma, qualis, tema/ objetivo de estudo e principais resultados encontrados.

Quadro 1 - Caracterização dos estudos incluídos na pesquisa. Rio de Janeiro, RJ, Brasil, 2020.

\begin{tabular}{|c|c|c|c|c|}
\hline $\begin{array}{l}\text { Autor(es)/ Local/ } \\
\text { Ano de publicação }\end{array}$ & Título & $\begin{array}{l}\text { Tipo de estudo/ } \\
\text { Idioma/ } \\
\text { Qualis }\end{array}$ & Tema/ objetivo & Principais resultados \\
\hline $\begin{array}{l}\text { Jarnulf, T.; } \\
\text { Skytt, B; } \\
\text { Martensson, G.; } \\
\text { Engstrom, M. } \\
\text { Suécia } \\
2019\end{array}$ & $\begin{array}{l}\text { District nurses } \\
\text { experiences of } \\
\text { precepting district } \\
\text { nurse students at } \\
\text { the postgraduate } \\
\text { level. }\end{array}$ & $\begin{array}{l}\text { Qualitativo. } \\
\text { Artigo em inglês. } \\
\text { A2 }\end{array}$ & $\begin{array}{l}\text { Descrever as experiências de } \\
\text { enfermeiras no ensino de } \\
\text { enfermeiras em nível de pós- } \\
\text { graduação. }\end{array}$ & $\begin{array}{l}\text { No nível de pós-graduação, altas } \\
\text { demandas são estabelecidas em } \\
\text { relação a conhecimentos, habilidades } \\
\text { e capacidade. }\end{array}$ \\
\hline $\begin{array}{l}\text { Filho, J.F.R.; } \\
\text { Santos, C.S. } \\
\text { Brasil } \\
2018\end{array}$ & $\begin{array}{l}\text { Identifying the } \\
\text { profiles and } \\
\text { activities of } \\
\text { preceptors in a } \\
\text { nursing residency } \\
\text { program. }\end{array}$ & $\begin{array}{l}\text { Quantitativo. } \\
\text { Artigo em inglês e } \\
\text { português. } \\
\text { B2 }\end{array}$ & $\begin{array}{l}\text { Identificar as atividades } \\
\text { desempenhadas pelos } \\
\text { preceptores da Residência em } \\
\text { Enfermagem do Hospital das } \\
\text { Clínicas em Pernambuco. }\end{array}$ & $\begin{array}{l}\text { Pouco engajamento dos preceptores } \\
\text { no planejamento das ações e } \\
\text { atividades a serem desempenhadas. } \\
\text { Percebeu-se falta de compreensão dos } \\
\text { preceptores sobre suas atividades e } \\
\text { funções no âmbito do Projeto } \\
\text { Pedagógico do Programa de } \\
\text { Residência. }\end{array}$ \\
\hline $\begin{array}{l}\text { Antunes, J.M.; } \\
\text { Daher, D.V.; } \\
\text { Ferrari, M.F.M. } \\
\\
\text { Brasil } \\
2017\end{array}$ & $\begin{array}{l}\text { Preceptoria como } \\
\text { lócus de } \\
\text { aprendizagem e } \\
\text { de coprodução de } \\
\text { conhecimento }\end{array}$ & $\begin{array}{l}\text { Qualitativo. } \\
\text { Artigo em } \\
\text { português. } \\
\text { B4 }\end{array}$ & $\begin{array}{ll}\text { Conhecer o processo } & \text { de } \\
\text { ensino-aprendizagem } & \text { de } \\
\text { preceptores de residentes } & \text { de } \\
\text { Enfermagem. } & \end{array}$ & $\begin{array}{l}\text { O papel da preceptoria é importante } \\
\text { na formação do residente de } \\
\text { Enfermagem, mesmo que haja } \\
\text { lacunas a serem revistas entre as } \\
\text { instituições de ensino e de saúde. A } \\
\text { aprendizagem que se efetiva entre } \\
\text { preceptores e residentes favorece a } \\
\text { socialização profissional, ampliando e } \\
\text { aprimorando os saberes apreendidos. }\end{array}$ \\
\hline $\begin{array}{l}\text { Delack, S.; } \\
\text { Martin, J.; } \\
\text { McCarthy, A.M.; } \\
\text { Sperhac, A.M. } \\
\\
\text { Iowa/ EUA } \\
2015\end{array}$ & $\begin{array}{l}\text { Nurse residency } \\
\text { programs and the } \\
\text { transition to child } \\
\text { health nursing } \\
\text { practice. }\end{array}$ & $\begin{array}{l}\text { Resumo. } \\
\text { Artigo em inglês. } \\
\text { Sem qualis. }\end{array}$ & $\begin{array}{l}\text { Discutir questões críticas } \\
\text { acerca dos programas de } \\
\text { residência em enfermagem } \\
\text { pediátrica. }\end{array}$ & $\begin{array}{l}\text { Embora a importância das residências } \\
\text { de enfermagem esteja bem } \\
\text { estabelecida, permanece } \\
\text { necessidade de articular os } \\
\text { componentes essenciais desses } \\
\text { programas. }\end{array}$ \\
\hline $\begin{array}{c}\text { Ribeiro, K.R.B.; } \\
\text { Prado, M.L. }\end{array}$ & $\begin{array}{l}\text { A prática } \\
\text { educativa dos } \\
\text { preceptores nas } \\
\text { residências em } \\
\text { saúde: um estudo } \\
\text { de reflexão. }\end{array}$ & $\begin{array}{l}\text { Estudo de } \\
\text { reflexão } \\
\text { Artigo em } \\
\text { português. } \\
\text { B1 }\end{array}$ & $\begin{array}{l}\text { Refletir sobre o exercício da } \\
\text { preceptoria como prática } \\
\text { educativa na formação e } \\
\text { qualificação do profissional } \\
\text { em saúde para o sistema único } \\
\text { de saúde. }\end{array}$ & $\begin{array}{l}\text { Para ensinar, é necessário } \\
\text { conhecimentos para além do conteúdo } \\
\text { da disciplina e, considerando que a } \\
\text { preceptoria é uma prática de educação } \\
\text { no trabalho, o preceptor precisa de } \\
\text { formação pedagógica. }\end{array}$ \\
\hline Wiseman, R.F. & Survey of & Estudo descritivo & Determinar as barreiras e os & As escolas de enfermagem lutam com \\
\hline
\end{tabular}




\begin{tabular}{|c|c|c|c|c|}
\hline $\begin{array}{c}\text { Maryland/ EUA } \\
2013\end{array}$ & $\begin{array}{l}\text { advanced practice } \\
\text { student clinical } \\
\text { preceptors. }\end{array}$ & $\begin{array}{c}\text { Artigo em inglês. } \\
\text { Sem qualis. }\end{array}$ & $\begin{array}{l}\text { motivadores para fornecer } \\
\text { orientação e avaliação clínica. } \\
\text { Determinar a necessidade de } \\
\text { preparação educacional para } \\
\text { facilitar seus papéis como } \\
\text { preceptores clínicos. }\end{array}$ & $\begin{array}{l}\text { a escassez de preceptores de prática } \\
\text { avançada. Cada escola de } \\
\text { enfermagem precisará encontrar } \\
\text { maneiras de reconhecer e oferecer } \\
\text { recompensas e incentivos simbólicos } \\
\text { que sejam viáveis dentro de suas } \\
\text { limitações econômicas. }\end{array}$ \\
\hline $\begin{array}{l}\text { Haggerty, C; } \\
\text { Holloway, K.; } \\
\text { Wilson, D. } \\
\text { Nova Zelândia } \\
\quad 2013\end{array}$ & $\begin{array}{l}\text { How to grow our } \\
\text { own: an } \\
\text { evaluation of } \\
\text { preceptorship in } \\
\text { New Zealand } \\
\text { graduate nurse } \\
\text { programmes. }\end{array}$ & $\begin{array}{c}\text { Estudo } \\
\text { longitudinal } \\
\text { Artigo em inglês. } \\
\text { A2 }\end{array}$ & $\begin{array}{l}\text { Fornecer informações sobre as } \\
\text { lições aprendidas, identificar e } \\
\text { compartilhar fatores de } \\
\text { sucesso e apoiar o } \\
\text { desenvolvimento das melhores } \\
\text { práticas a nível nacional. }\end{array}$ & $\begin{array}{l}\text { A disponibilidade e o preparo dos } \\
\text { preceptores para sua função geravam } \\
\text { altos níveis de satisfação para os } \\
\text { preceptores, residentes e outros } \\
\text { enfermeiros da área clínica. }\end{array}$ \\
\hline $\begin{array}{l}\text { Barbeiro, F.M.S.; } \\
\text { Miranda, L.V.; } \\
\text { Souza, S.R. } \\
\text { Brasil } \\
2010\end{array}$ & $\begin{array}{l}\text { Enfermeiro } \\
\text { preceptor e } \\
\text { residente de } \\
\text { enfermagem: a } \\
\text { interação no } \\
\text { cenário da } \\
\text { prática. }\end{array}$ & $\begin{array}{c}\text { Artigo em } \\
\text { português. } \\
\text { B1 }\end{array}$ & $\begin{array}{l}\text { Identificar o sentido atribuído } \\
\text { às relações entre preceptor e } \\
\text { residente e analisar como as } \\
\text { representações sociais se } \\
\text { fazem presentes na relação } \\
\text { entre enfermeiro preceptor e } \\
\text { residente. }\end{array}$ & $\begin{array}{l}\text { O relacionamento entre residente e } \\
\text { preceptor normalmente é visto como } \\
\text { uma relação professor/aluno, embora } \\
\text { o residente possua conhecimentos } \\
\text { mais atualizados. A troca de } \\
\text { conhecimentos se dá, geralmente, do } \\
\text { preceptor para o residente. }\end{array}$ \\
\hline
\end{tabular}

Fonte: Autores (2020).

Conforme descrito no Quadro 1, o tipo de metodologia mais utilizada pelos artigos selecionados foi de caráter qualitativo com um total de três pesquisas $(37,5 \%)$, seguido de um (12,5\%) estudo quantitativo, um (12,5\%) longitudinal, um $(12,5 \%)$ descritivo, um $(12,5 \%)$ de reflexão e um $(12,5 \%)$ resumo.

\section{Discussão}

Os resultados deste estudo permitiram identificar as contribuições e desafios vivenciados pelos enfermeiros preceptores dos Programas de Residência em Enfermagem. Na maioria dos artigos incluídos nesta revisão, o papel do preceptor apresentou carências no que se refere às qualificações, competências e reconhecimento profissional.

Estudo americano apontou a necessidade de aperfeiçoamento dos preceptores. Segundo os enfermeiros participantes, os preceptores precisam de educação em áreas específicas para o desenvolvimento de novos conhecimentos, habilidades e obtenção de maior variedade de métodos de ensino (Delack et al., 2015). Demogalski et al. (2021) destacam que a qualificação dos preceptores, com embasamento pedagógico, trata-se de uma ferramenta facilitadora ao ensino clínico e à resolutividade de demandas teórico-práticas dos residentes da área da saúde.

Estudo neozelandês afirma que a sobrecarga de trabalho dos enfermeiros reduz as chances de participação em treinamentos e capacitações de preceptoria (Haggerty et al., 2013), resultando no exercício de preceptores sem formação na área da educação (Lima, Almeida, Rangel, \& Pinheiro, 2020) e despreparados para o desenvolvimento da função (Jarnulf $e t$ al., 2019).

Ainda sobre a qualificação dos preceptores de enfermagem, estudo brasileiro destaca maior prevalência de profissionais com especialização nos moldes de residência e em menor frequência, titulações de mestrado. Os autores complementam que existe uma lacuna entre as unidades de ensino e de treinamento em serviço com pouca participação dos preceptores no planejamento das ações, nas atividades a serem realizadas e no projeto pedagógico do programa, dificultando a definição de objetivos e metas educacionais (Filho \& Santos, 2018). A fragilidade da integração entre as instituições de ensino e os serviços de saúde evidenciam uma necessidade de estreitamento entre o campo da prática e da teoria para a compreensão do papel do preceptor (Ribeiro \& Prado, 2013; Antunes et al., 2017).

Estudo revelou que enfermeiros com especialização nos moldes de residência apresentam melhor relacionamento com 
os residentes de enfermagem por conhecerem as dificuldades vivenciadas e as particularidades do processo de ensinoaprendizagem (Barbeiro et al., 2010). A maneira de conduzir a preceptoria está implícita diretamente às experiências e práticas de trabalho dos preceptores. Investigação americana constatou que grande parte dos enfermeiros relatam ensinar da mesma forma como foram ensinados por seus preceptores (Wiseman, 2013). Outros observam o desempenho dos residentes e oferecem feedback positivo ou negativo, objetivando a aprendizagem construtiva e a socialização profissional. Embora cientes do escopo estabelecido pela universidade, empenham-se na identificação dos propósitos individuais, guiando a construção da identidade profissional dos alunos (Jarnulf et al., 2019; Filho \& Santos, 2018; Antunes et al., 2017).

A atribuição de tarefas e a assunção de determinadas responsabilidades inerentes ao cuidado de enfermagem, baseiam-se no nível de conhecimento e habilidades dos residentes, com aumento gradual a partir da independência dos alunos, que devem ser avaliados constantemente pelos preceptores através de observações, diálogos e reflexões conjuntas (Jarnulf et al., 2019). Porém, estudo brasileiro aponta prática inadequada de preceptores que delegam suas atividades para os residentes, que acabam desenvolvendo-as sem o devido auxílio e supervisão (Barbeiro et al., 2010).

Martins e Scarella (2020) apresentam projeto pedagógico para a preceptoria de enfermeiros considerando que a sistematização das ações de preceptoria corrobora com a melhoria das atividades inerentes à formação dos residentes, além de estímulo e motivação dos preceptores. Ressaltam ainda que uma equipe de preceptores organizada, com metas e objetivos educacionais bem delimitados, favorecem o desenvolvimento de um perfil profissional pautado no conhecimento técnicocientífico, crítico e reflexivo, capaz de identificar, compreender e solucionar problemas reais do cenário de trabalho. Tal projeto pedagógico intitulado "Plano de desenvolvimento de habilidades e competências por Step's para estágio em unidade de terapia intensiva cardiológica" é composto por etapas de progressão e níveis de complexidade de acordo com o desenvolvimento de competências e habilidades relacionadas à prática clínica.

Como elementos que prejudicam o desenvolvimento do processo de preceptoria nos serviços de saúde pública, citamse dificuldades estruturais e administrativas nas instituições de saúde comprometendo o desenvolvimento de muitas ações do enfermeiro preceptor; pouca sensibilização da própria gestão do serviço quanto à relevância da preceptoria; acréscimo de mais uma função ao rol de atividades que os preceptores já realizam cotidianamente; não receber nenhuma remuneração adicional ou dias de folga por desempenharem este papel (Antunes et al., 2017; Wiseman, 2013), negativa de liberação institucional para participação em atividades de aperfeiçoamento e ausência de acesso às capacitações (Silva et al., 2021).

Silva et al. (2021) complementam que o cotidiano do trabalho dos enfermeiros preceptores contribui para o afastamento da vida acadêmica e atualizações em decorrência de impossibilidade de se ausentar do serviço pelas demandas profissionais, cansaço e falta de tempo, e que a interação com os estudantes estimula a busca por aperfeiçoamento e novos saberes. Salientam que o educador precisa ser incentivado e amparado pelas instituições de ensino, atendendo as necessidades dos profissionais, permitindo o empoderamento dos preceptores para participação ativa nos eixos de treinamento em serviço e acadêmico.

Preceptores de um Programa de Residência Multiprofissional do estado do Paraná (Brasil) referiram como aspectos relacionados à melhoria da qualidade do curso de especialização: maior efetividade na integração com residentes, tutores, professores, coordenadores e gestores dos programas e fortalecimento das relações interpessoais; comunicação efetiva entre residentes, preceptores e membros do corpo docente, com reuniões técnicas para discussão e planejamento de diretrizes, orientações, estratégias e cronogramas; adequação entre as demandas práticas e a formação acadêmica; capacitação profissional dos residentes; e capacitação dos preceptores destinadas à formação para preceptoria (Demogalski et al., 2021).

Estudo realizado com profissionais da saúde que concluíram um curso de especialização para preceptores do Sistema Único de Saúde (SUS), identificou que a participação na atividade acadêmica favoreceu reflexões sobre metodologias de ensino, com enfoque nas metodologias ativas, mudança do conceito tradicional de formação, modelo de educação centrado nas 
práticas problematizadoras tendo o aluno como sujeito ativo no processo de ensino, novas possibilidades para as práticas pedagógicas, amadurecimento profissional e a figura do preceptor como facilitador do processo de aprendizagem (ChiancaNeves, Lauer-Leite, \& Priante, 2020).

Para conseguir gerir de forma eficiente o tempo e responder à produtividade exigida pelo gestor, alguns preceptores optam por executar procedimentos técnico-assistenciais em detrimento de ensinar e orientar os residentes que estão sob sua supervisão (Antunes et al., 2017). As altas demandas para o cumprimento de metas diárias, execução das tarefas específicas à área da saúde, exaustão emocional, baixa realização profissional, somadas as atividades de preceptoria, constituem-se em risco de adoecimento dos enfermeiros preceptores por doenças específicas ao ambiente social de desempenho das atividades laborais, como por exemplo, a síndrome de burnout (Delack et al., 2015).

Apesar dos desafios e dificuldades descritas os enfermeiros preceptores salientam que a formação que se efetiva nos campos da prática contribui para o processo de ensino-aprendizagem com a possibilidade de coproduzir saberes e agregar novas experiências. Configura ainda, para o preceptor, o compromisso e a responsabilidade na construção do conhecimento em saúde, como ator relevante para o processo de formação dos residentes (Antunes et al., 2017), auxiliando na educação, socialização e qualificação profissional.

\section{Considerações Finais}

Este estudo permitiu identificar que a preceptoria nos Programas de Residência em Enfermagem exige dos enfermeiros altas demandas de conhecimentos teóricos e habilidades práticas, formação pedagógica para o exercício da preceptoria e aproximação com o campo acadêmico para participação nos programas de especialização e desenvolvimento da prática da educação no trabalho.

O processo de trabalho do enfermeiro preceptor envolve a rotina institucional dos cenários de trabalho, somados ao acompanhamento e supervisão ativa na formação dos residentes de enfermagem, transformando a vivência do campo profissional em experiências de aprendizagem e aperfeiçoamento. Porém, os preceptores alegam falta de reconhecimento, recompensas ou incentivos simbólicos que desestimulam o desempenho dos preceptores dos programas de especialização. Mesmo insatisfeitos e sobrecarregados entendem que a preceptoria contribui para a formação dos alunos e busca constante de novos saberes.

Conclui-se que se faz necessário investimento na qualificação profissional dos preceptores na área do ensino, atuação conjunta com as unidades de ensino no planejamento pedagógico, clareza sobre as atribuições e importância do preceptor na educação dos residentes, assim como retribuição através de remuneração e/ou oferta de benefícios aos profissionais que desempenham esta função. Fatores contribuintes à satisfação, realização profissional e desenvolvimento de competências teórico-práticas do enfermeiro preceptor.

Sugere-se estudos futuros, no campo de trabalho, para ampliação do conhecimento da atuação do enfermeiro preceptor e reflexão sobre as dificuldades e necessidades expressas por esses profissionais, permitindo movimento de construção coletiva de novas práticas, baseadas em problematizações reais, e que proporcionem melhorias das condições de trabalho, instrumentalização de competências e habilidades e participação ativa no planejamento e acompanhamento acadêmico.

\section{Referências}

Antunes, J. M., Daher, D. V., \& Ferrari, M. F. M. (2017). Preceptoria como lócus de aprendizagem e de coprodução de conhecimento. Rev enferm UFPE online, 111(10):3741-3748. https://doi.org/10.5205/1981-8963-v11i10a22612p3741-3748-2017

Barbeiro, F. M. S., Miranda, L. V., \& Souza, S. R. (2010). Enfermeiro preceptor e residente de enfermagem: a interação no cenário da prática. R. pesq.: cuid. fundam. Online, 2(3):1080-1087. 
Brasil (1996). Conselho Federal de Enfermagem. Anteprojeto de Lei $\mathrm{n}^{\circ}$ 2.264/1996. Dispõe sobre a Residência em Enfermagem e a sua respectiva Comissão Nacional. Rio de Janeiro.

Brasil (2001). Conselho Federal de Enfermagem - COFEN. Resolução nº 259/2001, de 12 de julho de 2001. Estabelece padrões mínimos para registro de Enfermeiro Especialista, na modalidade de Residência em Enfermagem.

Chianca-Neves, M. G. B., Lauer-Leite, I. D., \& Priante, P. T. (2020). As concepções de preceptores do SUS sobre metodologias ativas na formação dos profissionais da saúde. Educação em Revista, 36:e207303. http://dx.doi.org/10.1590/0102-4698207303

Delack, S., Martin, J., McCarthy, A. M., \& Sperhac, A. M. (2015). Nurse residency programs and the transition to child health nursing practice. J Nurs Adm, 45(6):345-50. http://dx.doi.org/10.1097 / NNA.0000000000000210

Demogalski, J. T., Alves, F. B. T., Cabral, L. P. A., Grden, C. R. B., Bordin, D., \& Fadel, C. B. (2021). Qualificação da residência multiprofissional em saúde: opiniões críticas de preceptores. Rev Fund Care Online, 13:136-143. http://dx.doi.org/10.9789/2175-5361.rpcfo.v13.7974

Fajardo, A. P., Rocha, C. M. F., \& Pasini, V. L. (2010). Grupo Hospitalar Conceição. Residências em saúde: fazeres e saberes na formação em saúde. Porto Alegre: Hospital Nossa Senhora da Conceição.

Ferreira, F. D. C., Dantas, F. C., \& Valente, G. S. C. (2018). Nurses' knowledge and competencies for preceptorship in the basic health unit. Rev Bras Enferm, 71(Suppl 4):1564-1571. http://dx.doi.org/10.1590/0034-7167-2016-0533

Filho, J. F. R., Santos, C. S. (2018). Identifying the Profiles and Activities of Preceptors in a Nursing Residency Program. O Mundo da Saúde, 42(2):333-348. http://dx.doi.org/10.15343/0104-7809.20184202333348

Haggerty, C., Holloway, K., \& Wilson, D. (2013). How to grow our own: an evaluation of preceptorship in New Zealand graduate nurse programmes. Contemp Nurse, 43(2):162-71. http://dx.doi.org/10.5172/conu.2013.43.2.162.

Jarnulf, T., Skytt, B., Martensson, G., \& Engstrom, M. (2019). District nurses experiences of precepting district nurse students at the postgraduate level. Nurse Education in Practice, 37:75-80. https://doi.org/10.1016/j.nepr.2019.05.004

Lima, P. O., Almeida, H. F., Rangel, S. C., \& Pinheiro, M. L. P. (2020). Preceptoria em enfermagem: contribuições e desafios. Revista Vozes dos Vales UFVJM, 18(10):1-20

Martins, G. M., Caregnato, R. C. A., Barroso V. L. M., \& Ribas, D. C. P. (2016). Implementação de residência multiprofissional em saúde de uma universidade federal: trajetória histórica. Rev Gaúcha Enferm, 37(3):e57046. http://dx.doi.org/10.1590/1983-1447.2016.03.57046.

Martins, L. S., \& Scarcella, M. F. S. (2020). Elaboração de projeto pedagógico para preceptoria de enfermeiros em terapia intensiva cardiológica. Revista Nursing, 23(269):4695-4698. https://doi.org/10.36489/nursing.2020v23i269p4695-4702

Paczek, R. S., \& Alexandre, E. M. (2019). Preceptoria em enfermagem em um serviço público de saúde. Rev enferm UFPE online. 13:e242697. https://doi.org/10.5205/1981-8963.2019.242697

Ribeiro, K. R. B., \& Prado, M. L. (2014). A prática educativa dos preceptores nas residências em saúde: um estudo de reflexão. Rev Gaúcha Enferm., 34(4):161-165. http://dx.doi.org/10.1590/1983-1447.2014.01.43731

Silva, V. C., Viana, L. O., Rasche, A. S., Aperibense, P. G. G. S., Telles, A. C., \& Matias, D. O. (2021). Capacitação para o exercício da preceptoria pelo enfermeiro na Residência Multiprofissional em Saúde. Revista Eletrônica Acervo Saúde, 13(3):e7017. https://doi.org/10.25248/reas.e7017.2021

Wiseman, R. F. (2013). Survey of advanced practice student clinical preceptors. J Nurs Educ, 52(5):253-258. https://doi.org/10.3928 / 01484834-20130319-03

Whittemore, R. (2005). Combining evidence in nursing research: methods and implications. Nursing Research, 54(1):56-62. https://doi.org/10.1097/00006199-200501000-00008

Zanoni, C. S., Haddad, M. C. L., Rossaneis, M. A., Vannuchi, M. T. O., \& Gvozd, R. (2015). Contribuições da residência em enfermagem na atuação profissional de egressos. Semina: Ciências Biológicas e da Saúde, 36(1):215-224. http://dx.doi.org/10.5433/1679-0367.2015v36n1Suplp215 